\title{
LMI Approach to Output Feedback Stabilization for Fuzzy Systems with Immeasurable Premise Variables
}

\author{
Jun YONEYAMA*
}

\begin{abstract}
This paper is concerned with output feedback control design for a fuzzy system with immeasurable premise variables. It is well known that Takagi-Sugeno fuzzy model describes a wide class of nonlinear systems especially when its premise variables include immeasurable functions. However, when it comes to control design of such a fuzzy system with immeasurable premise variables, a conventional parallel distributed compensator (PDC) is not feasible because it shares the same premise variables as those of a fuzzy system. In this paper, we introduce an output feedback controller with the estimate of the premise variables of an original fuzzy system. We then formulate the stabilization problem for a fuzzy system with immeasurable premise variables. Our control design method is based on a set of strict LMI conditions. No tuning parameter is necessary a priori to solve LMI conditions. Our method includes tuning matrices for control gains in a controller and hence they can be chosen to optimize the control performance of the system. Numerical examples are finally given to illustrate our control design method.
\end{abstract}

Keywords : Takagi-Sugeno fuzzy systems, Output feedback control, Premise variables

\section{Introduction}

It is well-known that the Takagi-Sugeno fuzzy model is one of the effcient mathematical models that describe a wide class of nonlinear systems ([5], [9]). Hence, its analysis and control synthesis are important. In the past years, suffcient stability conditions were given, and control design methods were pro$\operatorname{posed}([6],[10],[11],[12])$. A conventional approach to control design for a fuzzy system is to employ a parallel distributed compensator (PDC). It is shown that a PDC controller works well for a fuzzy system because it shares the same premise variables as those of the original fuzzy system. The papers [6] and [12] gave stability conditions and proposed a control design method by a PDC controller. Furthermore, relaxed stability conditions have been investigated in [10] and [11]. These results were only concerned with the state feedback control design, and the premise variables were assumed to be available. However, this is not always the case in many situations. In practical situations, the state of the system is not measurable and we need an output feedback control

* Department of Electrical Engineering and Electronics, Aoyama Gakuin University design. If the premise variable is the state, a conventional PDC controller, which feeds back the state variables, is infeasible. Furthermore, when the premise variable is the state, the Takagi-Sugeno model describes the widest class of nonlinear systems. A conventional PDC controller can work only for a limited class of nonlilnear systems ([14]).

In this paper, we propose a design method of a stabilizing output feedback controller for a fuzzy system with immeasurable premise variables. Such a fuzzy system describes a large class of nonlinear systems. The results in [3], [7], [8], [14] do not take care of this class of fuzzy systems. Yoneyama et al. [14] gave an approximate stabilizing output feedback controller. Assawinchaichote et al. [1] and Yoneyama [13] proposed an uncertain fuzzy system approach. Guerra et al. [2] gave a design method based on non-strict LMI conditions. These results are conservative and are not easy to solve controller design conditions. Here, our method is based on strict LMI conditions. We give a two-step procedure to solve the design problem. No tuning parameter is required in the control design, which is much easier than others in the literature. In addition, we have free scaling matrices that can tune control gain matrices. Finally, some 
numerical examples are given to illustrate our design procedure and the effectiveness of our design method.

\section{Fuzzy Systems}

In this section, we introduce Takagi-Sugeno fuzzy systems. Consider the Takagi-Sugeno fuzzy model, described by the following IF-THEN rules:

$$
\begin{array}{ll}
\text { IF } & \xi_{1} \text { is } M_{i 1} \text { and } \cdots \text { and } \xi_{p} \text { is } M_{i p}, \\
\text { THEN } & \dot{x}(t)=A_{i} x(t)+B_{i} u(t), \\
& y(t)=C_{i} x(t), \quad i=1, \cdots, r
\end{array}
$$

where $x(t) \in \Re^{n}$ is the state, $u(t) \in \Re^{m}$ is the control input and $y(t) \in \Re \Re^{q}$ is the measurement output. The matrices $A_{i}, B_{i}$ and $C_{i}$ are constant matrices of appropriate dimensions. $r$ is the number of IF-THEN rules. $M_{i j}$ are fuzzy sets and $\xi_{1}, \cdots, \xi_{p}$ are premise variables. We set $\xi=\left[\xi_{1} \cdots \xi_{p}\right]^{T}$. The premise variable $\xi(t)$ is assumed to be a function of the state, which is immeasurable.

For simplicity, we define the following notations:

$$
\begin{gathered}
X_{\xi}=\sum_{i=1}^{r} \lambda_{i}(\xi(t)) X_{i}, \quad X_{\hat{\xi}}=\sum_{i=1}^{r} \lambda_{i}(\hat{\xi}(t)) X_{i}, \\
X_{\xi \hat{\xi}}=\sum_{i=1}^{r} \sum_{j=1}^{r} \lambda_{i}(\xi(t)) \lambda_{j}(\hat{\xi}(t)) X_{i j},
\end{gathered}
$$

and so on.

Then, the state equation and the output equation are described, respectively, by

$$
\begin{aligned}
\dot{x}(t) & =\sum_{i=1}^{r} \lambda_{i}(\xi)\left\{A_{i} x(t)+B_{i} u(t)\right\} \\
& \triangleq A_{\xi} x(t)+B_{\xi} u(t), \\
y(t) & =\sum_{i=1}^{r} \lambda_{i}(\xi) C_{i} x(t) \triangleq C_{\xi} x(t)
\end{aligned}
$$

where

$$
\lambda_{i}(\xi)=\frac{\beta_{i}(\xi)}{\sum_{i=1}^{r} \beta_{i}(\xi)}, \quad \beta_{i}(\xi)=\prod_{j=1}^{p} M_{i j}\left(\xi_{j}\right)
$$

and $M_{i j}(\cdot)$ is the grade of the membership function of $M_{i j}$. We assume

$$
\beta_{i}(\xi(t)) \geq 0, i=1, \cdots, r, \sum_{i=1}^{r} \beta_{i}(\xi(t))>0
$$

for any $\xi(t)$. Hence $\lambda_{i}(\xi(t))$ satisfy

$$
\lambda_{i}(\xi(t)) \geq 0, i=1, \cdots, r, \sum_{i=1}^{r} \lambda_{i}(\xi(t))=1
$$

for any $\xi(t)$.

As stated in [13], [14], a fuzzy system covers a wider class of nonlinear systems when its premise variables are the state variables than when they are the measurement outputs. In order to design a stabilizing controller, a PDC controller is traditionally used for stabilization because it shares the same premise variables as those of a fuzzy system([6], [12]). However, a PDC controller is feasible only if the premise variables are measurable or available functions, Thus, most existing results have assumed that the premise variables are measurable ([3], [7], [8], [14]). In this paper, we consider an output feedback stabilization where the state is not directly measurable. A fuzzy system is supposed to have the premise variables that are functions of the states, which are immeasurable. In this case, actual values of the premise variables are not available. Hence, we employ the following rules for an output feedback controller with the premise variables being their estimates:

$$
\begin{array}{ll}
\text { IF } & \hat{\xi}_{1} \text { is } M_{i 1} \text { and } \cdots \text { and } \hat{\xi}_{p} \text { is } M_{i p}, \\
\text { THEN } & \hat{\dot{x}}(t)=\hat{A}_{i} \hat{x}(t)+\hat{B}_{i} y(t), \\
& u(t)=\hat{C}_{i} \hat{x}(t), \quad i=1, \cdots, r
\end{array}
$$

where $\hat{A}_{i}=\sum_{j=1}^{r} \lambda_{j}(\hat{\xi}(t)) \hat{A}_{i j}$, and $\hat{A}_{i j}, \hat{B}_{i}$ and $\hat{C}_{i}$ are constant matrices to be determined. Then the natural choice of a controller is given by

$$
\begin{aligned}
\dot{\hat{x}}(t) & =\sum_{i=1}^{r} \sum_{j=1}^{r} \lambda_{i}(\hat{\xi}) \lambda_{j}(\hat{\xi})\left\{\hat{A}_{i j} \hat{x}(t)+\hat{B}_{j} y(t)\right\} \\
& \triangleq \hat{A}_{\hat{\xi} \hat{\xi}} \hat{x}(t)+\hat{B}_{\hat{\xi}} y(t), \\
u(t) & =\sum_{i=1}^{r} \lambda_{i}(\hat{\xi}) \hat{C}_{i} \hat{x}(t) \triangleq \hat{C}_{\hat{\xi}} \hat{x}(t) .
\end{aligned}
$$

The closed-loop system (1) with (2) is given by

$$
\dot{x}_{e}(t)=\bar{A}_{\xi \hat{\xi} \hat{\xi}} x_{e}(t)
$$

where $x_{e}=\left[\begin{array}{ll}x^{T} & \hat{x}^{T}\end{array}\right]^{T}$ and

$$
\bar{A}_{\xi \hat{\xi} \hat{\xi}}=\left[\begin{array}{cc}
A_{\xi} & B_{\xi} \hat{C}_{\hat{\xi}} \\
\hat{B}_{\hat{\xi}} C_{\xi} & \hat{A}_{\hat{\xi} \hat{\xi}}
\end{array}\right] .
$$

Our problem is to find an output feedback controller (2) that stabilizes the closed-loop system (3).

The following lemma is useful to prove our main results.

Lemma 2.1 ([11])

$\sum_{i=1}^{r} \sum_{j=1}^{r} \lambda_{i}(\xi(t)) \lambda_{j}(\xi(t)) \Phi_{i j}<0$

holds if the followings are satisfied: 
$\begin{aligned} \Phi_{i i}<0, & i=1, \cdots, r, \\ \frac{2}{r-1} \Phi_{i i}+\Phi_{i j}+\Phi_{j i}<0, & i, j=1, \cdots, r, i \neq j\end{aligned}$

\section{Stability and Stabilization}

Here, we consider an output feedback control of a fuzzy system. We first give sufficient conditions for the closed-loop system (3) to be stable. Then, we propose a design method of an output feedback controller for a fuzzy system.

\subsection{Stabilization Analysis}

We make the stability analysis of the closed-loop system (3).

Theorem 3.1 An output feedback controller of the form (2) stabilizes the fuzzy system (1) if there exist matrices $X>0, Y>0, L_{\hat{\xi} \hat{\xi}}, W_{\hat{\xi}}, Z_{\hat{\xi}}$ such that

$$
\begin{aligned}
& {\left[\begin{array}{cc}
Y & I \\
I & X
\end{array}\right]>0,} \\
& \Psi_{\xi \hat{\xi} \hat{\xi}}=\left[\begin{array}{cc}
\Psi_{11 \xi \hat{\xi}} & \Psi_{12 \xi \hat{\xi} \hat{\xi}} \\
\Psi_{12 \xi \hat{\xi} \hat{\xi}}^{T} & \Psi_{22 \xi \hat{\xi}}
\end{array}\right]<0
\end{aligned}
$$

where

$$
\begin{aligned}
\Psi_{11 \xi \hat{\xi}}= & A_{\xi} X+X A_{\xi}^{T}+B_{\xi} Z_{\hat{\xi}}+Z_{\hat{\xi}}^{T} B_{\xi}^{T}, \\
\Psi_{12 \xi \hat{\xi} \hat{\xi}}= & A_{\xi}+X A_{\xi}^{T} Y+X C_{\xi}^{T} W_{\hat{\xi}}^{T}+Z_{\hat{\xi}}^{T} B_{\xi}^{T} Y \\
& +L_{\hat{\xi} \hat{\xi}}^{T}, \\
\Psi_{22 \xi \hat{\xi}}= & Y A_{\xi}+A_{\xi}^{T} Y+W_{\hat{\xi}} C_{\xi}+C_{\xi}^{T} W_{\hat{\xi}}^{T} .
\end{aligned}
$$

Proof: We investigate the stability of the closed-loop system (3). A condition for the system (3) to be stable is that there exists a matrix $\mathrm{P}>0$ such that

$\bar{A}_{\xi \hat{\xi} \hat{\xi}}^{T} P+P \bar{A}_{\xi \hat{\xi} \hat{\xi}}<0$.

To solve the matrix inequality (6), we follow the idea of [4] and partition $P$ and $P^{-1}$ as

$P=\left[\begin{array}{cc}Y & N \\ N^{T} & V\end{array}\right], \quad P^{-1}=\left[\begin{array}{cc}X & M \\ M^{T} & U\end{array}\right]$,

respectively. Define a transformation matrix $\Pi$ as

$\Pi=\left[\begin{array}{cc}X & I \\ M^{T} & 0\end{array}\right]$.

Then, it can be inferred from $P P^{-1}=I$ that

$Y X+N M^{T}=I$.

We take a congruence transformation with $\Pi$ for $P$ to obtain
$\Pi^{T} P \Pi=\left[\begin{array}{cc}X & I \\ I & Y\end{array}\right]>0$,

which is exactly the same as (4). It follows that $Y-$ $X^{-1}>0$ and hence $N M^{T}=I-Y X$ is nonsingular.

We take a congruence transformation with $\Pi$ for (6) to obtain

$$
\Pi^{T} \bar{A}_{\xi \hat{\xi} \hat{\xi}}^{T} P \Pi+\Pi^{T} P \bar{A}_{\xi \hat{\xi} \hat{\xi}} \Pi<0,
$$

or more exactly we get (5) where we define

$L_{\hat{\xi} \hat{\xi}}=N \hat{A}_{\hat{\xi} \hat{\xi}} M^{T}, W_{\hat{\xi}}=N \hat{B}_{\hat{\xi}}$, and $Z_{\hat{\xi}}=\hat{C}_{\hat{\xi}} M^{T}$.

\subsection{Stabilization}

This section gives sufficient conditions for the closed-loop system (3) to be stable, and a method of controller design base on such stability conditions.

Theorem 3.2 An output feedback controller of the form (2) stabilizes the fuzzy system (1) if there exist matrices $X>0, Y>0, L_{j k}, W_{j}, Z_{j}, j, k=1, \cdots, r$ such that (4) holds and

$$
\begin{aligned}
\Psi_{i j j}<0, & i, j=1, \cdots, r, \\
\frac{2}{r-1} \Psi_{i j j}+\Psi_{i j k}+\Psi_{i k j}<0, & i=1, \cdots, r, \\
& j \neq k=1, \cdots, r
\end{aligned}
$$

where

$$
\begin{aligned}
\Psi_{i j k}= & {\left[\begin{array}{cc}
\Psi_{11 i j} & \Psi_{12 i j k} \\
\Psi_{12 i j k}^{T} & \Psi_{22 i j}
\end{array}\right], } \\
\Psi_{11 i j}= & A_{i} X+X A_{i}^{T}+B_{i} Z_{j}+Z_{j}^{T} B_{i}^{T}, \\
\Psi_{12 i j k}= & A_{i}+X A_{i}^{T} Y+X C_{i}^{T} W_{j}^{T}+Z_{j}^{T} B_{i}^{T} Y \\
& +L_{j k}^{T}, \\
\Psi_{22 i j}= & Y A_{i}+A_{i}^{T} Y+W_{j} C_{i}+C_{i}^{T} W_{j}^{T},
\end{aligned}
$$

In this case, control gain matrices in (2) are given by

$$
\begin{aligned}
& \hat{A}_{j k}=N^{-1} L_{j k} M^{-T}, \\
& \hat{B}_{j}=N^{-1} W_{j}, \\
& \hat{C}_{j}=\bar{Z}_{j} M^{-T}, i, j, k=1, \cdots, r,
\end{aligned}
$$

where $M$ and $N$ can be found from the equation $N M^{T}=I-Y X$.

Proof: If conditions (13) are satisfied, it follows from Lemma 2.1 that

$\sum_{i=1}^{r} \sum_{j=1}^{r} \sum_{k=1}^{r} \lambda_{i}(\xi(t)) \lambda_{j}(\hat{\xi}(t)) \lambda_{k}(\hat{\xi}(t)) \Psi_{i j k}=\Psi_{\xi \hat{\xi} \hat{\xi}}<0$.

By Theorem 3.1, the stability is established. Control gain matrices (14) follow from the relations (12). 
Theorem 3.2 does not give LMI conditions. However, the following algorithm provides an efficient LMIbased approach to solve for (4) and (13).

Algorithm 3.1 Step 1: Solve $Y>0, W_{j}, j=1, \cdots, r$ for the following LMI conditions

$\Psi_{22 i j}<0, i, j=1, \cdots, r$.

Step 2: Insert matrices $Y, W_{j}, j=1, \cdots, r$ which are obtained in Step 1 into $\Psi_{i j k}$. Then, solve $X>0, L_{j k}, Z_{j}$, $j, k=1, \cdots, r$ for (4) and (13).

Step 3: If there exists a solution, obtain gain matrices as in (14).

Remark 3.1 One way to find $M$ and $N$ is that we let $M=\alpha$ I to obtain $N=(I-Y X) / \alpha$ where $\alpha$ is some scalar. $M$ and $N$ can tune control gain matrices in (2), which reflect trajectories of the states of the controlled system.

Remark 3.2 Applying the transformation with $\tilde{x}_{e}=$ $\Pi x_{e}$ to the system (3), we have

$\dot{\tilde{x}}_{e}(t)=\tilde{A}_{\xi \hat{\xi} \xi} \tilde{x}_{e}(t)$

where $\widetilde{A}_{\xi \hat{\xi} \hat{\xi}}=\Pi^{-1} \bar{A}_{\xi \hat{\xi} \hat{\xi}} \Pi$. Since (11) can be written as

$\tilde{A}_{\xi \hat{\xi} \hat{\xi}}^{T} \tilde{P}+\tilde{P} \tilde{A}_{\xi \hat{\xi} \hat{\xi}}<0$

where $\widetilde{P}=\Pi^{T} P \Pi$, the existence of $\tilde{P}>0$ in (16) with (10) guarantees the stability of (15). By definition of $\tilde{x}_{e}, \tilde{x}_{e}$ can be calculated as

$\tilde{x}_{e}=\left[\begin{array}{cc}0 & M^{-T} \\ I & -X M^{-T}\end{array}\right]\left[\begin{array}{l}x \\ \hat{x}\end{array}\right]$.

If we choose $M=X$, then we have

$\tilde{x}_{e}=\left[\begin{array}{c}X^{-1} \hat{x} \\ x-\hat{x}\end{array}\right] \triangleq\left[\begin{array}{c}X^{-1} \hat{x} \\ e\end{array}\right]$.

In this case, the estimate $\hat{x}$ and the error e go to zero as tgoes to infinity. It implies that $\hat{x}$ in the controller (2) works as an observer which estimates the state variables, and it also converges to zero.

Remark 3.3 Assawinchaichote et al. [1] and Yoneyama [13] considered the grade $\lambda_{i}(\xi)$ as uncertainty, and then gave stability conditions. Since their results depend on the maximum upper bound of $\lambda(\xi)$, they are rather conservative. The result of Guerra et al. [2] is based on non-LMI conditions with four scaling parameters, which must be chosen a priori. Algorithm 3.1 is involved with only strict LMI conditions, which are much easier to be solved than other methods. Unlike the approach in [4], Algorithm 3.1 provides a two-step algorithm. This is due to the more complicated structure of a controller (2) than that of a linear controller.

\section{Examples}

We give two examples. The first one is given in [2] and compares with the result in [2]. The second example is a general nonlinear control system. We note that the results in $[3],[7],[8],[14]$ cannot be applied to these problems.

Example 4.1 We take the same example in [2]. Consider the system (1) where $\lambda_{1}\left(x_{1}\right)=0.5+\arctan \left(x_{1}\right) /$ $\pi, \lambda_{2}\left(x_{1}\right)=1-\lambda_{1}\left(x_{1}\right)$, and

$$
\begin{aligned}
& A_{1}=\left[\begin{array}{cc}
1 & 0 \\
-1 & -1
\end{array}\right], A_{2}=\left[\begin{array}{cc}
2.5 & 0 \\
-2.3 & -1
\end{array}\right], \\
& B_{1}=B_{2}=\left[\begin{array}{l}
1 \\
0
\end{array}\right], C_{1}=C_{2}=\left[\begin{array}{ll}
10 & 2
\end{array}\right] .
\end{aligned}
$$

We attempt to obtain an output feedback controller of the form (2). In this problem, the output $y$ is the only measurable and premise variable $x_{1}$ is immeasurable. Algorithm 3.1 guarantees the existence of an output feedback controller. When we choose $M=I$, a controller (2) is given with

$$
\begin{aligned}
& \hat{A}_{11}=\left[\begin{array}{cc}
-15.1566 & 1.1214 \\
-1.1904 & -0.3592
\end{array}\right] ; \\
& \hat{A}_{12}=\left[\begin{array}{cc}
-16.0809 & 4.4549 \\
-1.4356 & -0.3966
\end{array}\right] \text {, } \\
& \hat{A}_{21}=\left[\begin{array}{cc}
-25.3805 & -1.1346 \\
-1.7777 & -0.4945
\end{array}\right] \text {, } \\
& \hat{A}_{22}=\left[\begin{array}{cc}
-15.2504 & 1.0588 \\
-1.2009 & -0.3648
\end{array}\right], \\
& \hat{B}_{1}=\left[\begin{array}{l}
0.0651 \\
0.0015
\end{array}\right], \hat{B}_{2}=\left[\begin{array}{l}
0.0652 \\
0.0014
\end{array}\right] \text {, } \\
& \hat{C}_{1}=\left[\begin{array}{ll}
-104.2435 & 77.8624
\end{array}\right], \\
& \hat{C}_{2}=\left[\begin{array}{ll}
-105.0520 & 77.4877
\end{array}\right] \text {. }
\end{aligned}
$$

When considering Remark 3.2 we choose $M=X$, a controller (2) is given with

$$
\begin{aligned}
& \hat{A}_{11}=\left[\begin{array}{cc}
-16.5702 & -2.7484 \\
8.8236 & 1.0543
\end{array}\right], \\
& \hat{A}_{12}=\left[\begin{array}{cc}
-16.6707 & -2.0857 \\
7.669 & 0.1932
\end{array}\right],
\end{aligned}
$$




$$
\begin{gathered}
\hat{A}_{21}=\left[\begin{array}{cc}
-28.6671 & -5.4000 \\
16.7729 & 2.7921
\end{array}\right], \\
\hat{A}_{22}=\left[\begin{array}{cc}
-16.6840 & -2.7809 \\
8.8696 & 1.069
\end{array}\right], \\
\hat{B}_{1}=\left[\begin{array}{c}
1.2965 \\
-1.0471
\end{array}\right], \hat{B}_{2}=\left[\begin{array}{c}
1.2964 \\
-1.0470
\end{array}\right], \\
\hat{C}_{1}=\left[\begin{array}{ll}
-5.3004 & -0.1993
\end{array}\right], \\
\hat{C}_{2}=\left[\begin{array}{ll}
-5.3521 & -0.2125
\end{array}\right] .
\end{gathered}
$$

Simulation results of $x_{1}$ and $\hat{x}_{1}$ with the initial conditions $x(0)=\left[\begin{array}{lll}100 & 100\end{array}\right]^{T}, \hat{x}(0)=\left[\begin{array}{ll}0 & 0\end{array}\right]^{T}$ for the above two controllers are depicted in Figures 1 and 2, respectively. Both of them stabilize the system. But, Figure 1 shows the observer does not work well to estimate the state due to low gains $\hat{B}_{i}, i=1,2$, while Figure 2 indicates that the observer works and the controller makes a shorter setting time and smaller undershoot. Compared with the result in Guerra et al [2], the second controller provides a better control performance in Figure 2. In other words, Algorithm 3.1 proposes not only a stabilizing controller but also tuning matrices $N$ and $M$ for the control performance.

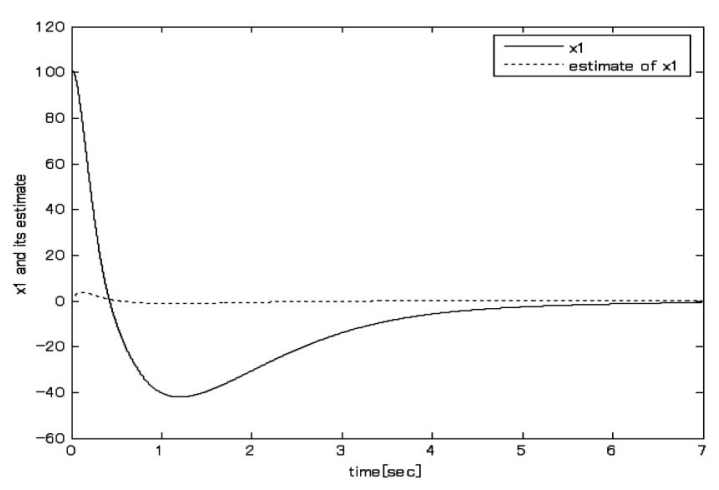

Figure 1 State and its estimate

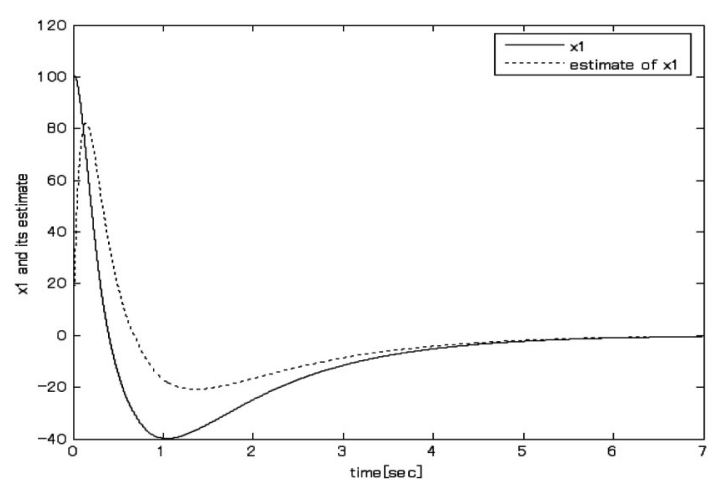

Figure 2 State and its estimate
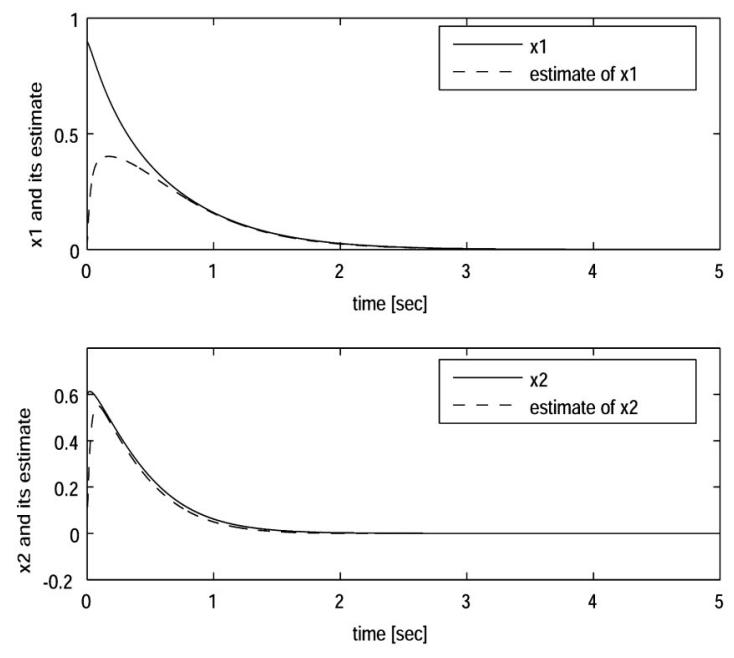

Figure 3 States and their estimates

Example 4.2 Next, we consider a nonlinear system:

$$
\begin{aligned}
\dot{x}_{1} & =-2 x_{1}+0.3 x_{1} x_{2}+x_{2}+0.5 x_{1} u, \\
\dot{x}_{2} & =0.2 x_{1}^{2}+x_{2}+u, \\
y & =0.1 x_{1}^{2}+2 x_{2}
\end{aligned}
$$

Assuming $x_{1} \in[-11]$, we can write the above system as a fuzzy system (1) where $\xi=x_{1}, \lambda_{1}\left(x_{1}\right)=\left(-x_{1}+\right.$ 1) $/ 2, \lambda_{2}\left(x_{1}\right)=\left(x_{1}+1\right) / 2$ and

$$
\begin{aligned}
& A_{1}=\left[\begin{array}{cc}
-2 & 0.7 \\
-0.2 & 2
\end{array}\right], A_{2}=\left[\begin{array}{cc}
-2 & 1.3 \\
0.2 & 2
\end{array}\right], \\
& B_{1}=\left[\begin{array}{c}
-0.5 \\
1
\end{array}\right], B_{2}=\left[\begin{array}{c}
0.5 \\
1
\end{array}\right], \\
& C_{1}=\left[\begin{array}{ll}
-0.1 & 2
\end{array}\right], C_{2}=\left[\begin{array}{ll}
0.1 & 2
\end{array}\right] .
\end{aligned}
$$

This fuzzy system has the premise variable $x_{1}$, which is immeasurable.

Algorithm 3.1 with $M=\left[\begin{array}{cc}45 & -32 \\ -6 & 10\end{array}\right]$ gives an output feedback controller (2) with $\hat{\xi}=\hat{x}_{1}$ and

$$
\begin{gathered}
\hat{A}_{11}=\left[\begin{array}{cc}
9.4814 & 21.9602 \\
-13.4613 & -25.5181
\end{array}\right], \\
\hat{A}_{12}=\left[\begin{array}{cc}
119.1479 & 151.6085 \\
-111.1490 & -143.6439
\end{array}\right], \\
\hat{A}_{21}=\left[\begin{array}{cc}
-103.0612 & -105.6591 \\
83.3711 & 86.5725
\end{array}\right], \\
\hat{A}_{22}=\left[\begin{array}{cc}
9.09150 & 21.4095 \\
-13.1191 & -25.02617
\end{array}\right], \\
\hat{B}_{1}=\left[\begin{array}{cc}
-14.7586 \\
18.1742
\end{array}\right], \hat{B}_{2}=\left[\begin{array}{c}
-14.7590 \\
18.1732
\end{array}\right], \\
\hat{C}_{1}=\left[\begin{array}{ll}
-1.2254 & -1.6913
\end{array}\right], \\
\hat{C}_{2}=\left[\begin{array}{ll}
-1.2175 & -1.6785
\end{array}\right] .
\end{gathered}
$$


Simulation results with the initial conditions $x(0)=$ $\left[\begin{array}{ll}0.8 & 0.6\end{array}\right]^{T}, \hat{x}(0)=\left[\begin{array}{ll}0 & 0\end{array}\right]^{T}$ are shown in Figure 3. It is clear that the obtained controller stabilizes the system.

\section{Conclusions}

We have proposed an output feedback controller for fuzzy systems with immeasurable premise variables. Our design method was based on LMI approach. No tuning parameter is necessary to solve the output feedback controller design problem. It also gives not only a stabilizing controller but also is concerned with the control performance. Finally, some numerical examples are given to illustrate our method.

\section{References}

[ 1 ] W. Assawinchaichote, S.K. Nguang, and P. Shi, Fuzzy Control and Filter Design for Uncertain Fuzzy Systems, Springer, 2006.

[2 ] T.M. Guerra, A. Kruszewski, L. Vermeiren, and H. Tirmant, Conditions of output stabilization for nonlinear models in the Takagi-Sugeno's form, Fuzzy Sets and Systems, Vol.157, pp.1248-1259, 2006.

[ 3 ] X.J. Ma, Z.Q. Sun, and Y.Y. He, Analysis and design of fuzzy controller and fuzzy observer, IEEE Transactions on Fuzzy Systems, Vol.6, No.1, pp.41-50, 1998.

[ 4 ] C. Scherer, P. Gahinet, and M. Chilali, Multiobjective output-feedback control via LMI optimization, IEEE Transactions on Automatic Control, Vol.42, No.7, pp.896-911, 1997.

[ 5 ] T. Takagi and M. Sugeno, Fuzzy identification of systems and its applications to modeling and control, IEEE Transaction on Systems, Man, Cybernetics, Vol.15, pp.116-132, 1985.

[6 ] K. Tanaka and M. Sugeno, Stability analysis and design of fuzzy control systems, Fuzzy Sets and Systems, Vol.45, pp.135-156, 1992.
[ 7 ] K. Tanaka and M. Sano, On the concepts of regulator and observer of fuzzy control systems, 3rd IEEE International Conference on Fuzzy Systems, pp.767-772, 1994.

[ 8 ] K. Tanaka, T. Ikeda, and H.O. Wang, Fuzzy regulators and fuzzy observers: relaxed stability conditions and LMI-based designs, IEEE Transactions on Fuzzy Systems, Vol.6, No.2, pp.1-16, 1998.

[ 9 ] K. Tanaka and H.O. Wang, Fuzzy Control Systems Design and Analysis: A Linear Matrix Inequality Approach, Wiley Inter-Science, 2000.

[10] M.C.M. Teixeira, E. Assuncao and R.G. Avellar, On relaxed LMI- based designs for fuzzy regulators and fuzzy observers, Vol.11, pp.613-623, 2003.

[11] H.D. Tuan, P. Apkarian, T. Narikiyo and Y. Yamamoto, Parameterized linear matrix inequality techniques in fuzzy control system design, IEEE Transactions on Fuzzy Systems, Vol.9, pp.324-332, 2001.

[12] H.O. Wang, K. Tanaka, and M. Griffin, An approach to fuzzy control of nonlinear system stability and design issues, IEEE Transactions on Fuzzy Systems, Vol.4, No.1, pp.14-21, 1996.

[13] Jun Yoneyama, $\mathrm{H}_{\infty}$ output feedback control for fuzzy systems with immeasurable premise variables: discrete-time case, Applied Soft Computing, Vol.8, No,2, pp.949- 958, 2008.

[14] J. Yoneyama, M. Nishikawa, H. Katayama and A. Ichikawa, Design of output feedback controllers for Takagi-Sugeno fuzzy systems, Fuzzy Sets and Systems, Vol.121, No.1, pp.127-148, 2001.

(2009年11月21日 受付)

(2010年 4 月 2 日 採録)

[Contact Address]

5-10-1 Fuchinobe, Chuo-ku, Sagamihara, Kanagawa 2525258, JAPAN

Department of Electrical Engineering and Electronics, Aoyama Gakuin University

Jun YONEYAMA

TEL : 042-759-6527

E-mail : yoneyama@ee.aoyama.ac.jp

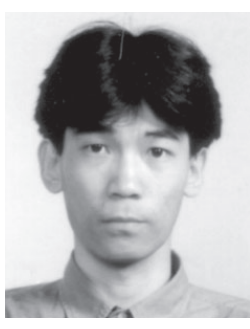

Jun YONEYAMA [member]

Received the B.Eng. and M.Eng. degrees in electrical engineering from Waseda University, Tokyo, Japan. He obtained Ph.D. degree in electrical engineering from University of California, Los Angles in 1996. Currently, he is an Associate Professor in Department of Electrical Engineering and Electronics in Aoyama Gakuin University, Tokyo, Japan. His current research interest includes fuzzy systems, time-delay systems, robust control, stochastic control, sampled-data control, and their applications. 